\title{
Assessment of Uptake of Free Maternity Services among Women Visiting Kisumu East District Hospital, Kisumu County, Kenya
}

\author{
Article by Chepkemoi Judy ${ }^{1}$ and Taratisio Ndwiga ${ }^{2}$ \\ ${ }^{1}$ Department of Environmental Health, School of Public Health, Moi University, \\ KENYA \\ ${ }^{2}$ School of Public Health, Texila American University, KENYA \\ Email: taratisiondwiga@yahoo.com, thandichjudy@gmail.com
}

\begin{abstract}
Introduction: Maternity services are an essential part in any and all communities. This includes care from a traditional birth attendants or a trained $H \mathrm{~W}$. This is aimed at improving the maternal health and reducing child mortality which in the long run will lead to development.

Problem statement: There has been a problem of overcrowding and poor quality of services already in public hospitals before the introduction of free maternity. This new change saw a rise in the number of women delivering in the hospital increase drastically causing a strain in the already tight situation. Many women hence prefer to deliver delivering in the hands of traditional birth attendants and only coming to the hospital if and when complications occur. This has led to the high mortality and maternal morbidity rates

Justification: Maternity is a crucial part in any and all societies in ensures continuity from generation to generation. It is important to ensure that all women of child bearing age can easily access the services from a skilled birth attendant.

Objectives: Broad: To assess the Uptake of free maternity services among women visiting Kisumu East District Hospital. Kisumu East District Hospital was selected for the research due to its convenience and proximity to the researcher. Descriptive study design was chosen that seeks to describe the pattern of a phenomena by person, place and time. Convenience sampling was used by drawing a representative data. People were selected because of the ease of their volunteering and units because of their availability of easy access.

Data collection: Data was collected by administration of questionnaires where key informants were interviewed. Analysis: SPSS version 20.0 used for data analysis.

Findings: Ninety four percent 94\% of the respondents were aware of the free maternity services $93 \%$ of the women visited ANC clinic during their last pregnancy while only $7 \%$ did not. A total of $76 \%$ of the respondents delivered at home while the rest $24 \%$ delivered in hospital. The most common reason for delivering at home among the women was accessibility to a health facility at $41 \%$.

Conclusion: Most mothers were aware of the free maternity services available in Kenya hence attended attend ante-natal and post-natal clinics. Although they are aware of the services available, many of the mothers do not deliver in the hospital due to the low standards of the services caused by overcrowding, low stuffing and insufficient supply of medication.

Recommendation: Undertake a comprehensive maternal health education campaign which focuses on key causes of delay of treatment and seeks to promote gender equality in health decision-making to allocate more funds to the free maternity services implementation to improve its implementation and improve the quality of services provided in the health facilities.
\end{abstract}

Keywords: Free maternity services, maternal morbidity and mortality rates, Antenatal care and maternal deaths

\section{Introduction}

Kenya has long suffered from high maternal morbidity and mortality rates. The most recent estimates set the maternal mortality rate at 488 deaths per 100,000 live births, well above the 
MDG target of 147 per 100,000 by 2015 . The problem is driven, at least in part, by lack of access to quality maternal health services, including ante-natal, delivery, and post-natal services. Reproductive health is widely recognized to include family planning, Antenatal, delivery, and postnatal health services. The Kenyan Constitution of 2010 further provides that a person has the right to emergency treatment (Article 43(2), the right to inherent dignity and the right to have that dignity respected and protected (Article 28), and the right to access information (Article 35). The Kenya National Patients 'Rights Charter (2013) outlines the right to access health care, the right to receive emergency treatment in any health facility irrespective of ability to pay, the right to the highest attainable quality of health care products and services, the right to be treated with respect and dignity, the right to information, and the right to complain, among others. This move by President Uhuru Kenyatta will improve maternal health, as most mothers will now deliver under skilled care, said Dr. Francis Kimani, the Director of Medical Services (HERAF, 2013). The decision to waiver maternity fees was also aimed at reducing the Kenya's high maternal death rate.

\section{Problem statement}

There has been overcrowding and poor quality of services already in public hospitals before the introduction of free maternity. This new change saw a rise in the number of women delivering in the hospital increase drastically causing a strain in the already tight situation. This has caused a burden to the service providers where seven nurses are allocated to 4000 residents which half the recommended 14 nurses per 4000residents is leading to the decrease in the quality of services provided. Many women hence prefer to deliver in the hands of traditional birth attendants and only coming to the hospital if and when complications occur. This has led to the high mortality and maternal morbidity rates of 488 deaths per 100,000 live births which is well above MDG target of 147 deaths per 100,000 by 2015 (Bourbonnaise, 2013).

\section{Justification}

Maternity is a crucial part in any and all societies and ensures continuity from generation to generation. It is hence important to ensure that all women of child bearing age can easily access the services from a skilled birth attendant. The framework for free maternal health services is provided for under several local, regional, and international mandates. Article 43(1) (a) of the Constitution of Kenya 2010 states that: Every person has the right to the highest attainable standard of health, which includes the right to health care services, including reproductive health care. About $78 \%$ were home deliveries (last childbirth) even after visiting the ANC (Antenatal care).

\section{Research questions and objectives}

Research questions included was 'What is the uptake of free maternity services among women visiting Kisumu East District Hospital' while the broad objective was to assessment of the uptake of the free maternity services among women visiting Kisumu East District Hospital, with Specific objectives, to assess the knowledge on free maternity services, to establish the level of uptake of free maternity services among women visiting postnatal clinic in Kisumu East District Hospital and to determine the challenges faced by the women when accessing the free maternity services.

\section{Literature review}

\section{Knowledge of the free maternity}

If pregnant women have the knowledge on kinds of services offered by public hospital the value of implementing these services will be realized while if people do not utilize the service then it doesn't make any economic sense. Also the condition of facilities ought to be at its best to attract more patients to use the services while at the same time all deliveries should be 
with the assistance from trained healthcare worker who is capable of identifying the signs of complication and act appropriately (Griffiths and Stephenson 2001).

Many women have the correct knowledge regarding minimum numbers of antenatal visits to be done by a pregnant woman (WHO guideline). Nearly two-fifth participants knew schedule of antenatal care (ANC) visits. Almost $60 \%$ had done $\geq 4$ ANC visits during last pregnancy. Majority visited Sub Health Post/Health Post/Primary Health Care Centre for ANC Checkup. Gyawali, 2013 explains that while up to 92 percent of expectant mothers in Kenya attend antenatal care, more than half of them do not deliver in hospitals. In some regions such as Kenya's Nyanza and Western provinces, about 75 percent of women do not deliver in hospital, (Gyawali, 2013).

\section{Level of uptake of free maternity services among women visiting postnatal clinic in kisumu east district hospital}

Despite China's rapid economic growth, maternal and infant health in rural areas remains a policy concern. There are a large number of new programs in the area of public health: folic acid for pregnant women; programs to encourage delivery in hospitals; immunization programs for new-borns; and initiatives to increase health awareness and literacy on issues ranging from prenatal to postnatal and postpartum. Despite these initiatives, there is evidence that maternal and child health care service utilization remains low.

Giving birth with the help of a trained professional is critical in reducing maternal mortality, which can simply be defined as the death of a woman while pregnant or within 42 days of termination, from a cause related to the pregnancy or provoked by the pregnancy or its management. It is shocking to know that more than half of Kenyan women still give birth at home without the help of qualified attendants. According to the Kenya Demographic and Health Survey 2008/2009, 56 per cent of women give birth at home. The survey further says that 63 per cent of births in rural areas are delivered at home, But myths and misconceptions, as well as the lure of traditional birth attendants' supposed knowledge, are not the only reasons why expectant mothers shun hospital delivery.

\section{Challenges faced by the women when accessing the free maternity services}

Unfortunately, too often, pregnant women seeking maternity care receive varying degrees of ill treatment: from relatively subtle disrespect of their autonomy and dignity to outright abuse - physical assault, verbal insults, discrimination, abandonment, or detention in facilities for failure to pay. Evidence is now emerging that this fear of being badly treated and abused in health facilities is holding women back from seeking help. It is proving to be as big a deterrent as cost of care and transport. (Rowdon, 2014).

During the Madaraka day celebrations on June 1, 2013, President Uhuru Kenyatta announced the waiver of maternity charges. The news was received with delight by majority of Kenyans, particularly women. However, some were not too quick to celebrate as they sought to understand the reality of how that would work without compromising the quality of the services offered in these health institutions. In keeping with the promises made by the Jubilee Alliance during the election campaigns, the president said the scrapping of the fees would help all expectant mothers' access maternal care and would also help reduce maternal deaths.

The move by President Uhuru Kenyatta will improve maternal health, as most mothers will now deliver under skilled care, said Dr. Francis Kimani, the Director of Medical Services. This decision to waiver maternity fees was also aimed at reducing the Kenya's high maternal death rate. Although many mothers are taking advantage of the new free delivery services, some women still prefer the services of traditional birth attendants for various reasons. For some, it's because they live a long distance from the nearest health facility and because they do not trust hospitals. Antenatal consultations and facility-based deliveries are critical in improving the health of women and children. However, less than half of all pregnant women in Kenya receive the WHO-recommended minimum of four antenatal consultations, and only 
South American Journal of Public Health

Volume 4, Issue 2, 2016

43 percent of births occur in health facilities. Some of the reasons given by women for giving birth at home, particularly in the marginalised areas such as the North Eastern region of Kenya, seem valid. They argue that the health facilities are too far from where they live making it almost impossible to access hospital facilities (Bournnais, 2013).

PGH statistics show that only five percent of the population in the north-eastern region have access to health facilities for delivery. The remaining 95 percent rely on traditional birth as their only hope in ensuring safe delivery for both mother and child.

Physical access to health facilities through distance and/or lack of transport, and economic considerations are important barriers for women to delivering in a health facility in Kenya. Some women do not perceive a need to deliver in a health facility and may value health facility delivery less with subsequent deliveries. Access to appropriate transport for mothers in labour and improving the experiences and outcomes for mothers using health facilities at childbirth augmented by health education may increase uptake of health facility delivery in Kenya (Kitui, 2013). Women in public hospitals are either neglected by nurses or are attended to by medical trainees.

Health facilities are generally understaffed and in some regions, particularly in rural areas, you will find three or four nurses attending to children and the general public while at the same time delivering babies. Health experts have faulted the government's programme of free maternity services in public health facilities citing inadequate resources and personnel. It is quite evident from increase in the number of pregnant women seeking medical services in the public facilities that the public health facilities feel the burden of catering for an ever increasing number of patients i.e. the number of pregnant women has skyrocketed, leaving the nurses and other health workers lamenting about their workload. Further, it is noted that the pay, numbers and allowances of health workers has remained constant, resulting in poor motivation and consequently poor services delivery. While the maternity fee waiver is a progressive step, more needs to be done to care for Kenya's expectant mothers. Health facilities require more staff, and to be provided with the necessary drugs and equipment to provide maternity services (Ingati, 2013).

There are many more mothers who need help to deliver since the maternity waiver was introduced but the number of staff remains the same. Sometimes it becomes impossible to supervise the delivery of all mothers effectively hence a lot needs to be done to realise this all-important agenda of reducing maternal mortality in the country (Gathigah, 2015). Despite its commitment to maternal health care, Kenya continues to make slow progression with this regard. With a maternal mortality rate of 488 deaths per 100,000 live births, the country is off track in achieving the UN Millennium Development Goal numbers four and five by 2015 . Kenya had committed to reduce these deaths to 147 per 100,000 live births by 2015 .

\section{Methodology}

\section{Study area}

This research was carried out Kisumu East District Hospital which is a level Kisumu is a port city in Kisumu county, Kenya at 1,131 m (3,711 ft.), with a population of 409,928 (2009 census). It is the third largest city in Kenya, the principal city of western Kenya, the immediate former capital of Nyanza Province and the headquarters of County It is the largest city in Nyanza region. Kisumu East District Hospital is one of the hospitals in Kisumu County and attracts people from all over the region.

\section{Study design, target and sample population}

This research was a descriptive study and the target population were women of child bearing age accessing postnatal clinic services. The sample population were the women of childbearing age with the last child below the age of three years of age accessing postnatal clinic services. An average of 854 mothers that visit the postnatal clinic monthly. 


\section{Sample size determination}

Target population, $\mathrm{N}=854$ persons to represent the entire population of women accessing post-natal services in Kisumu East District Hospital Using Fisher's Formula. (Fisher et al, 1998).

The sample size was calculated using Fischer's method of epidemiological sample size determination where 128 was obtained.

Kisumu East District Hospital was purposefully chosen due to its proximity to us. The target population were women of child bearing age. They were conveniently chosen. Women with their last child below the age of three years of age were chosen.

\section{Data collection}

The tool had a set of questions which were administered by the interviewer to collect quantitative. The quantitative data that were collected using interviewers' schedule include; the age of the respondents, the knowledge of the respondents on free maternity services, the number of times the respondent have attended ANC, the number of hospital and home deliveries among others. To ensure uniformity in its administration and data collected, the interviewers' schedule was standardized.

Before administration of the tool, consent was sought from the respondent and confidentiality guaranteed.

Administration of the tool included the questions read to the respondent, clarification and interpretation made where necessary to the respondent and response was recorded quantitatively or qualitatively.

Key Informant Interview included seeking Verbal consent first, guarantying confidentiality to the key informant, addressed questions under various themes, recording the responses and picking the main points only.

\section{Data analysis procedures}

Data was analysed by the use of SPSS version 20.0. Frequencies and percentages were used to attain descriptive statistics and presented inform of tables, charts and graphs.

\section{Inclusive criteria and ethical clearance}

Inclusion criteria included women of reproductive age and Mothers with last child below the age 3 years. Permission was sought from the ethics department in the County as well as from the medical superintendent of the Kisumu East District Hospital.

\section{Results and findings}

\section{Demographic data}

A total of $54 \%$ of the respondents were below the age of 20 years with a percentage of $7 \%$ below the age of 15 years. Many of the respondents $82 \%$ were married while the minimum $3 \%$ were widowed. $86.9 \%$ of the respondents had attained primary education only while a minimum of $5.5 \%$ had attained tertiary education. $47 \%$ of those who were married were between the ages of 21-30 years while the lowest was between the ages of $16-20$ at $4.5 \%$.

Ninety four percent $(94 \%)$ of the respondents were aware of the free maternity services available while $6 \%$ were not aware. Ninety three percent $(93 \%)$ of the women visited ANC clinic during their last pregnancy while only $7 \%$ did not.

Forty six percent $(46 \%)$ of the women visited the ANC more than 4times which is the recommendation while the lowest number at $7 \%$ never visited the ANC.

A total of $78 \%$ of the respondents who visited the antenatal clinic during their last pregnancy delivered at home while the rest $22 \%$ delivered in hospital. 


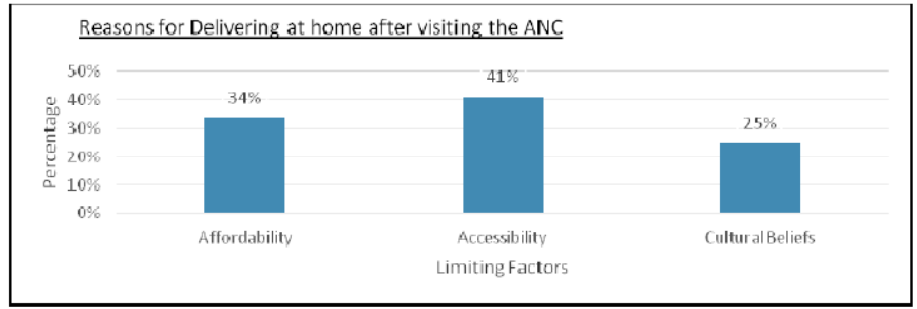

Reasons for Home Delivery

The most common reason for delivering at home among the women was accessibility to a health facility at $41 \%$ while the least was cultural beliefs at $25 \%$.

\section{Challenges faced when accessing the free maternity services.}

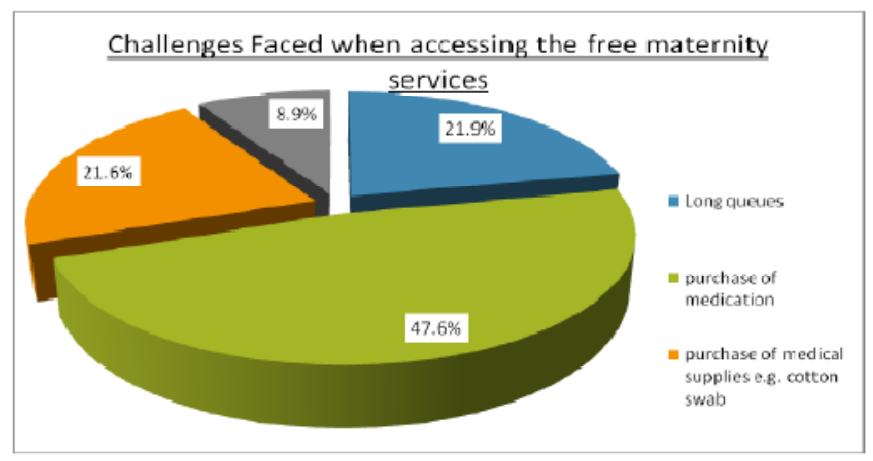

Challenges Faced by the Women when accessing the free maternity Service.

Forty seven percent $(47.6 \%)$ of the respondents stated that they had to purchase their medication while $8.9 \% \%$ said that they were treated harshly by the service providers. $21.9 \%$ of the respondents waited in line for long before they could be attended to and twenty one percent $(21.6 \%)$ of the respondents were forced to buy medical supplies for example cotton swab, methylated spirit, syringes among others.

\section{Discussions, conclusion and recommendation}

\section{Knowledge of the women on the free maternity services}

A total of $54 \%$ of the respondents were below the age of 20 years, while $87 \%$ of the respondents had attained primary education only while a minimum of $5 \%$ had attained tertiary education. The low level of education and high numbers of early marriages may be directly linked to the high numbers of early pregnancies. Majority of the respondents were aware of the free maternity services. This is due to the active campaign and advertisement that has been going on in the country.

Uptake of free maternity services among women visiting postnatal clinic in Kisumu East District Hospital.

The low levels of hospital deliveries may be directly linked to the low quality of services, and inaccessibility of the facilities. This has hence seen a total of $76 \%$ of the respondents delivered at home while only $24 \%$ delivered in hospital.

Although $93 \%$ of the women visited ANC clinic during their last pregnancy only $7 \%$ did not. Out of the $93 \%$ that visited the ANC, only $22 \%$ of them delivered in the hospital. This is in line with a statement by (Bournnais, 2013) who stated that $75 \%$ of women in Nyanza do not deliver in hospitals. All the respondents were able to access the maternity services that were being provided. 


\section{Challenges faced by women when accessing the services}

The low supply of medication forced mothers to buy for them which in the long run made the service expensive. Inadequate supply of medication made the services expensive which is contrary to the free maternity goals. This hence lead to the decrease in the number of hospital deliveries over the years these low levels of hospital deliveries may also be directly linked to the low quality of services, and inaccessibility of the facilities. By the expectant mothers this is in concurrences with an article.

The high bed capacity and the low staffing lead to low quality of the service provided. This hence saw mothers opting for other alternatives of hospital delivery

Inadequate supply of medication made the services expensive which is contrary to the free maternity goals.

The low bed capacity and the low staffing lead to low quality of the service provided. This hence saw mothers opting for other alternatives of hospital delivery which is hence in concurrent with a statement by (Ingati, 2013)"Health facilities are generally understaffed and in some regions, you will find three or four nurses attending to children and the general public while at the same time delivering babies".

\section{Conclusion}

The study has shown that most mothers are aware of the free maternity services available in Kenya. This has hence seen many of the women taking advantage of these services despite the quality of the services available. Most of the mothers hence attend ante-natal and postnatal clinics. Many of the women do not deliver in hospitals even after visiting ANC. This is mainly as a result of the fact that they end up purchasing medication as they seek the services which makes the maternity services expensive. Although they are aware of the services available, many of the mothers do not deliver in the hospital as expected during the free maternity introduction. This is according to the results acquired during this research. This is as a result of the low standards of the services due to overcrowding, low stuffing, and insufficient supply of medication. Improvement of the free maternity services has to be handled from different angles; various factors are dependent on each other as shown in the diagram below.

\section{Recommendation}

To the county government

- The county government should conduct a mass mobilisation and education to inform the women on the importance of hospital delivery.

- Encouraging and promoting girl child education to reduce the number of early marriages and early pregnancies.

To the national government

- Undertake a comprehensive maternal health education campaign which focuses on key causes of delay of treatment and seeks to promote gender equality in health decision-making.

- To allocate more funds to the free maternity services implementation to improve its implementation and improve the quality of services provided in the health facilities.

\section{References}

[1.] Bourbonnaise, N (2013) Implementing Free Maternity Health Care in Kenya. Enhancing the Realisation of Your Rights, pp. 1-14.

[2.] Bournnais, N (2013) Impementing free maternal health care in Kenya. KNCHR.

[3.] Fisher A.A (1998) Handbook for family planning. Operation research design in Sampling, 40-45.

[4.] Gathigah, M (2015) Inter Press Services. Kenya's mothers shun free maternity health care.

[5.] Griffin s and Stevenson (2001), Utilization of Maternal Healthcare Services in India, Understanding regional differences. University of Maryland, USA 
South American Journal of Public Health

Volume 4, Issue 2, 2016

[6.] Gyawali, K (2013) Knowledge and Practices on maternal health care among mothers. Journal of The Scientific Society, 9-13.

[7.] HERAF (2013) policy Changes To benefit women. Ministry of Health implements free maternity services nationwide.

[8.] Ingati, S. (2013) Kenya's free maternity services popular, but challenges remain.

[9.] Kitui, J (2013) Factors influencing place of delivery for women in Kenya: an analysis of the Kenya demographic and health survey. BMC Pregnancy Childbirth, pp. 13-40.

[10.] Rowdon, C (2014) Respectful Maternity Care. Healthy Mothers Healthy World, pp. 12-20.

[11.] Government Of Kenya (2010) The Constitution of Kenya. National Council for law.

[12.] WHO (2014) Maternal Mortality in 2014. Maternal Mortality Ratios and Rates., 20-32.

[13.] Ouma (2010) Antenatal and Delivery Care in Rural Western Kenya: The Effect of Training Health Care Workers to Provide "Focused Antenatal Care". MOH Kenya

[14.] UNICEF (2008) Countdown to 2015: Tracking Progress in Maternal, Newborn and Child Survival: Report; New York: UNICEF.

[15.] Yashide Nakamara (2010) Maternal and Child Health Handbook in Japan. Osaka University. JMAJ 53(4)

[16.] Bosire Boniface (2013) Kenyan Hospital Slow to Comply with Waived Maternity Fee Directive "http:sabahionline.com 